\title{
ELX-02 Generates Protein via Premature Stop Codon Read-Through without Inducing Native Stop Codon Read-Through Proteins ${ }^{[\rrbracket}$
}

\author{
Daniel K. Crawford, Iris Alroy, Neal Sharpe, Matthew M. Goddeeris, and Greg Williams
}

Eloxx Pharmaceuticals, Inc., Waltham, Massachusetts

Received February 7, 2020; accepted April 30, 2020

\begin{abstract}
ELX-02 is a clinical stage, small-molecule eukaryotic ribosomal selective glycoside acting to induce read-through of premature stop codons (PSCs) that results in translation of full-length protein. However, improved read-through at PSCs has raised the question of whether native stop codon (NSC) fidelity would be impacted. Here, we compare read-through by ELX-02 in PSC and NSC contexts. DMS-114 cells containing a PSC in the TP53 gene were treated with ELX-02 and tested for increased nuclear p53 protein expression while also monitoring two other proteins for NSC readthrough. Additionally, blood samples were taken from healthy subjects pre- and post-treatment with ELX-02 (0.3-7.5 mg/kg). These samples were processed to collect white blood cells and then analyzed by western blot to identify native and potentially elongated proteins from NSC read-through. In a separate experiment, lymphocytes cultivated with vehicle or ELX-02 (20 and $100 \mu \mathrm{g} / \mathrm{ml}$ ) were subjected to proteomic analysis. We found that ELX-02 produced significant read-through of the PSC found in TP53 mRNA in DMS-114 cells, resulting in increased p53 protein expression and consistent with decreased nonsense-mediated
\end{abstract}

mRNA degradation. NSC read-through protein products were not observed in either DMS-114 cells or in clinical samples from subjects dosed with ELX-02. The number of read-through proteins identified by using proteomic analysis was lower than estimated, and none of the NSC read-through products identified with $>2$ peptides showed dose-dependent responses to ELX-02. Our results demonstrate significant PSC read-through by ELX-02 with maintained NSC fidelity, thus supporting the therapeutic utility of ELX-02 in diseases resulting from nonsense alleles.

\section{SIGNIFICANCE STATEMENT}

ELX-02 produces significant read-through of premature stop codons leading to full-length functional protein, demonstrated here by using the R213X mutation in the TP53 gene of DMS-114 cells. In addition, three complementary techniques suggest that ELX-02 does not promote read-through of native stop codons at concentrations that lead to premature stop codon read-through. Thus, ELX-02 may be a potential therapeutic option for nonsense mutation-mediated genetic diseases.

\section{Introduction}

Approximately $10-12 \%$ of all mutations are single-point mutations that lead to an in-frame UAA, UAG, or UGA codon in messenger ribonucleic acid (mRNA) (Krawczak et al., 2000; Mort et al., 2008), thereby introducing a stop signal. These "nonsense" mutations that result in premature stop codons (PSCs) are the cause of a significant number of genetic diseases (Linde and Kerem, 2008), including cystic fibrosis, cystinosis, Usher syndrome, primary ciliary dyskinesia, polycystic kidney disease, mucopolysaccharidosis I, and a variety of cancers (Bordeira-Carrico et al., 2012). Nonsense mutations are single-nucleotide changes that may occur in germline or somatic DNA, by inaccurate or inefficient pre-mRNA splicing, or in the context of improper RNA editing. The disease phenotypes caused by nonsense mutations are frequently

https://doi.org/10.1124/jpet.120.265595.

S This article has supplemental material available at jpet.aspetjournals.org. more severe than those caused by missense mutations because nonsense mutations can completely eliminate protein function or can produce a small protein fragment, which often yields a dominant negative effect with protein partners (Frischmeyer and Dietz, 1999; Miller and Pearce, 2014). Moreover, premature termination fails to remove all exon junction complexes from the mRNA, which signals nonsensemediated decay (NMD) machinery to degrade mRNA and further limit protein production (Celik et al., 2015). Curative therapies do not exist for most nonsense mutation diseases, and treatment options are needed beyond palliative care.

Premature translation termination can be overridden by small molecule intervention, allowing for read-through and restoration of full-length protein. As an example, aminoglycoside antibiotics can induce translational read-through by binding to the decoding site in the small subunit of the ribosome (Burke and Mogg, 1985; Moazed et al., 1986; Pokrovskaya et al., 2010; Prokhorova et al., 2017; Dabrowski et al., 2018; Sabbavarapu et al., 2018). This site normally

ABBREVIATIONS: $\beta 2 \mathrm{M}, \beta 2$ microglobulin AGO1 argonaute-1 Cp crossing point; CysC, cystatin C; DAPI, 4',6-diamidino-2-phenylindole; ELX-02, 6'-(R)-Methyl-5-O-(5-amino-5,6-dideoxy- $\alpha$-L-talofuranosyl)-paromamine sulfate; FDR, false discovery rate HEK293, human embryonic kidney 293 $\mathrm{MDH}$ 1, malate dehydrogenase 1; NMD, nonsense mediated decay; NSC, native stop codon; PBMC, peripheral blood mononuclear cells; PEP, posterior error probability; PSC, premature stop codon; VEGF-A, vascular endothelial growth factor A. 
monitors proper codon-anticodon interactions, and when these read-through molecules bind to the decoding site, they induce a conformational change in the ribosome, which stabilizes the "exo" confirmation of two conserved adenosine residues (Shalev and Baasov, 2014; Shulman et al., 2014). This change reduces the ability of the ribosome to discriminate between cognate and near-cognate aminoacyl-transfer RNAs (Fourmy et al., 1998) and increases the probability that translational read-through of PSCs will occur. This contrasts with native stop codons (NSCs), in which the ability to induce read-through is markedly diminished because an empty decoding site will be recognized by a preassembled termination complex made up of proteins, including Poly(A)-binding protein and eukaryotic polypeptide chain release factors 1 and 3 (Schuller and Green, 2018).

In limited short-term studies, aminoglycoside antibiotics have shown potential to induce translational read-through across PSCs, resulting in functional protein and clinical efficacy (Clancy et al., 2001; Wilschanski et al., 2003). Despite these promising results, these compounds have limited readthrough efficacy and cannot be used as long-term therapies because of the potential to induce nephrotoxicity and ototoxicity following uptake and accumulation in renal proximal tubules and cochlear hair cells, respectively (Prayle et al., 2010; O'Sullivan et al., 2017). Though nephrotoxicity is reversible through kidney tubule cell proliferation, aminoglycoside antibiotic-induced ototoxicity can lead to permanent hearing loss due to the inability of cochlear hair cells to regenerate. Therefore, for effective long-term treatment of nonsense mutation diseases, compounds are needed that augment PSC read-through but lack the toxicities typically associated with this class of compounds.

The present studies compare PSC and NSC translational read-through following treatment with ELX-02 [6'-(R)Methyl-5-O-(5-amino-5,6-dideoxy- $\alpha$-L-talofuranosyl)-paromamine sulfate, also known as NB124 or ( $S$ )-9 from Kandasamy et al. (2012)], a small-molecule eukaryotic ribosomal selective glycoside engineered to read-through PSCs without the toxicities of aminoglycoside antibiotics (Leubitz et al., 2019). A cancer cell line carrying a nonsense mutation within the TP53 gene was evaluated for PSC read-through, and fulllength p53 protein production was quantified following ELX02 treatment while also monitoring for NSC read-through protein products. Blood samples were also collected pre- and post-treatment in healthy volunteers treated with ELX-02 in a Phase 1 study for protein analysis to further explore whether ELX-02 induces NSC read-through. Finally, unbiased proteomic analysis of isolated human peripheral blood mononuclear cells (PBMCs) treated with ELX-02 was performed to evaluate differences in the read-through proteome. Such studies are important to understand the intended and potentially unintended read-through products following treatment with eukaryotic ribosomal selective glycosides.

\section{Materials and Methods}

\section{DMS-114 Small-Cell Lung Cancer Cell Line}

Cell Culture. The DMS-114 small-cell lung cancer cell line (catalog number CRL-2066; American Type Culture Collection, Manassas, VA) contains a point mutation of $\mathrm{C}>\mathrm{T}$ at nucleotide 637 of the TP53 gene, resulting in a UGA premature stop at codon 213 $(R 213 X)$, which leads to a truncated p53 protein that lacks tumor suppressor activity. Cells were incubated at $37^{\circ} \mathrm{C}$ with $5 \% \mathrm{CO}_{2}$ in Waymouth's MB 752/1 medium (catalog number 31220-023; Thermo Fisher, Carlsbad, CA), supplemented with $10 \%$ of non-heat-inactivated FBS (catalog number F2442-500mL; Sigma-Aldrich, St. Louis, MO) and 1\% PenStrep (catalog number 15140-122; Thermo Fisher). Compound treatments were performed in the same medium but without PenStrep addition. Vehicle ( $1 \%$ or $2 \%$ water) was run in parallel on every plate as a negative control. G418 $(0.75 \mathrm{mM})$, considered to be one of the strongest read-through inducers (Bidou et al., 2017), was also run in parallel on every plate as a positive control.

Immunofluorescence Assay. DMS-114 cells were cultured in 96well plates $(25,000$ cells/well) and then treated with compound (either vehicle, G418, or ELX-02 in an eight-point concentration response curve from 0.023 to $3 \mathrm{mM}$ ) in biologic triplicates starting 24 hours later. Edge wells were filled with water to avoid evaporation in the remaining wells of the plate. Cells were fixed 48 hours post-treatment with $4 \%$ paraformaldehyde for 15 minutes at room temperature. Blocking buffer containing $3 \%$ bovine serum albumin and $0.2 \%$ Triton $\mathrm{X}-100$ in PBS was applied, followed by incubation steps overnight at $4^{\circ}$ C with mouse anti-p53 primary antibody (DO-1) (1:200, catalog number sc-126; Santa Cruz Biotechnology, Dallas, TX) and goat anti-mouse secondary antibody (1:1000, catalog number A21236; Thermo Fisher). To stain cell nuclei, 4',6-diamidino-2-phenylindole (DAPI, catalog number D3571; Invitrogen, Carlsbad, CA) was diluted in PBS and used at a final concentration of $1 \mu \mathrm{g} / \mathrm{ml}$ in each well. Cells were imaged at $10 \times$ magnification. Immunofluorescence was detected by using high content imaging (INCell6000 analyzer; GE Healthcare Life Sciences, Pittsburg, PA), and an algorithm was developed to quantify staining. The number of DAPI nuclei were counted, and the mean density of p53 nuclear expression was quantified.

Western Blot Assay. DMS-114 cells were cultured in 12-well plates $(666,000$ cells/well) and then treated with compound (either vehicle, G418, or ELX-02 in an eight-point concentration response curve from 0.023 to $3 \mathrm{mM}$ ) in biologic duplicates starting 24 hours later. Cells were lysed in radioimmunoprecipitation assay buffer (catalog number 89900; Thermo Fisher) containing protease and phosphatase inhibitors (catalog number 78443; Thermo Fisher) 48 hours post-treatment. Once protein content was quantified by using the BCA protein determination kit (catalog number 23225; Thermo Fisher), $60 \mu \mathrm{g}$ of each sample was added into a lane of a 12\% Bis-tris gel (catalog number NP0342; Thermo Fisher). For p53, cystatin $\mathrm{C}$ (CysC), and Lamin B1, protein transfer was accomplished using a $0.2-\mu \mathrm{m}$ polyvinylidene difluoride membrane in the iBlot2 dry blotting system (catalog number IB24001; Thermo Fisher). For vascular endothelial growth factor A (VEGF-A), protein transfer was accomplished by wet blotting onto a $0.45-\mu \mathrm{m}$ polyvinylidene difluoride membrane using $1 \times$ NuPage transfer buffer (catalog number NP0006; Thermo Fisher) and 10\% methanol in demineralized water for 1 hour at $30 \mathrm{~V}$. After blocking with 5\% skimmed milk in Tris-buffered saline/ Tween 20 for 1 hour at room temperature, blots were probed with mouse anti-p53 primary antibody (DO-1) (1:200, catalog number sc126; Santa Cruz Biotechnology) and goat anti-mouse secondary antibody (1:10,000, catalog number 925-32210; Li-Cor Biotechnology, Lincoln, NE). As a loading control, blots were also probed with rabbit anti-Lamin B1 primary antibody (1:1000, catalog number ab133741; Abcam, Cambridge, MA) and goat anti-rabbit secondary antibody (1: 10,000, catalog number 925-68073; Li-Cor Biotechnology). Each primary and secondary antibody incubation step occurred overnight at $4^{\circ} \mathrm{C}$. Proteins from one housekeeping gene (CysC, rabbit primary antibody used at 1:1000, catalog number 24840S; Cell Signaling Technologies, Danvers, MA) and one gene showing high native readthrough (VEGF-A, rabbit primary antibody used at 1:1000, catalog number ab46154; Abcam) were chosen for quantifying cell lysates already generated to assess p53 PSC read-through. For these two proteins, goat anti-rabbit secondary antibody (1:10,000, catalog number 925-68073; Li-Cor Biotechnology) was used. Recombinant proteins for p53 (catalog number ab84768; Abcam), CysC (catalog number H5041; Sigma Aldrich), and VEGF-A (catalog number 
ab155740; Abcam) were run during assay development to establish the limits of detection and sensitivity for each protein. All blots were imaged using the LiCor Odyssey CLx and quantified using image Studio v5.2 (Li-Cor Biotechnology).

Reverse-Transcription Quantitative Polymerase Chain Reaction Assay. DMS-114 cells were cultured in 96-well plates $(40,000$ cells/well) and then treated with compound (either vehicle, G418, or ELX-02 in an eight-point concentration response curve from 0.023 to 3 $\mathrm{mM}$ ) in biologic duplicates starting 24 hours later. RNA was harvested 24 hours post-treatment by using the MagMAX-96 Total RNA Isolation Kit (catalog number AM1830; Ambion, Austin, TX), and samples from two wells of each condition were pooled to obtain sufficient RNA. cDNA was synthesized in a separate reaction by using reverse transcriptase (catalog number 4368814; Applied Biosystems, Foster City, CA). Amplification was performed using Taqman fast advanced master mix (catalog number 4444554; Applied Biosystems) in technical triplicates. The gene for $\beta$-actin $(A C T N B$, assay ID Hs01060665_g1 with VIC-MGB dye; Thermo Fisher) was selected for multiplexing with the gene for p53 (TP53, assay ID Hs01034249_m1 with FAM-MGB dye; Thermo Fisher). Steady state gene expression was measured with the LightCycler 480 (Roche, Basel, Switzerland), using standard cycling conditions. Relative TP53 expression was then calculated based on the $\Delta \Delta \mathrm{Cp}$ method, where $\mathrm{Cp}$ is the crossing point:

$$
\begin{gathered}
\Delta \mathrm{Cp}=\mathrm{Cp}_{T P 53}-\mathrm{Cp}_{A C T N B} \\
\Delta \Delta \mathrm{Cp}=\Delta \mathrm{Cp}_{E L X-02 / G 418}-\Delta \mathrm{Cp}_{\text {Vehicle }} \\
\text { Fold change TP53 expression }=2^{-\Delta \mathrm{Cp} .} .
\end{gathered}
$$

Data Analysis. Data were graphed and analyzed using GraphPad Prism 8 software. Concentration response curves were subjected to nonlinear regression analysis [log(agonist) vs. response - variable slope (four parameters)]. One-way ANOVA with Dunnett post-test was run to determine compound efficacy relative to vehicle, with significance threshold set as $P<0.05$.

\section{Clinical Samples}

Collection and Analysis. Blood samples were collected predose as well as at 24,48 , and 144 hours post-ELX-02 treatment (0.3$7.5 \mathrm{mg} / \mathrm{kg}$ ) from healthy male and female subjects as part of a randomized, double-blinded, placebo-controlled, single-ascending dose, phase 1 clinical trial (Leubitz et al., 2019). Blood samples were similarly collected from subjects receiving placebo. In total, samples were collected from 51 subjects. EL-001 (NCT02807961) was conducted at Tel Aviv Sourasky Medical Center, Clinical Research Center (Tel Aviv 6423916, Israel), and EL-006 (NCT03292302) was conducted at SGS Life Sciences Services, Clinical Pharmacology Unit (Antwerp, Belgium). The studies were conducted in compliance with the Declaration of Helsinki and with the International Conference on Harmonization Note for Guidance on Good Clinical Practice (CPMP/ ICH/135/95), including the archiving of essential documents. Local institutional review boards (study 001, Tel-Aviv Sourasky Medical Center Helsinki Committee, Tel-Aviv Yafor, Israel; study 006, Commissie Voor Medische Ethiek, ZNA Institutional Review Board, ZNA/ OCMW, Antwerp, Belgium) reviewed and approved the study protocols and the informed consent documents. All subjects provided written informed consent before any study-related procedures were performed.

All samples were immediately processed for white cell fraction separation by using Ficoll-Paque PLUS reagent (catalog number 17144002; GE Healthcare). The resulting PBMCs were lysed by using either whole-cell lysis buffer (50 mM Tris-HCl, pH 7.5, $150 \mathrm{mM} \mathrm{NaCl}$, $5 \mathrm{mM}$ EDTA, 1\% NP-40, 0.1\% SDS, 0.8\% Deoxycholate, $0.1 \mathrm{mM}$ phenylmethylsulfonyl fluoride, $1 \times$ protease inhibitors) or radioimmunoprecipitation assay buffer [50 mM Tris-HCl, pH 8.0, $150 \mathrm{mM} \mathrm{NaCl}$, $1.0 \%$ Igepal CA-630 (NP-40), 0.5\% sodium deoxycholate, $0.1 \%$ SDS,
$0.1 \mathrm{mM}$ phenylmethylsulfonyl fluoride, and $1 \times$ protease inhibitors]. Lysates were then snap frozen in liquid nitrogen and stored at $-80^{\circ} \mathrm{C}$ until all samples were collected.

Lysates were defrosted and protein extracts diluted (ratios of 1:10 and 1:20), and the protein concentration was measured by using the Bradford assay reagent (catalog number 5000006; Bio-Rad Laboratories, Hercules, CA) with bovine serum albumin as a calibration standard. Crude extracts $(5-10 \mu \mathrm{g})$ were mixed with Laemmli buffer with $\beta$-mercaptoethanol as a reducing agent to a final concentration of $1 \times$. Extracts were analyzed by western blot for the expression of two housekeeping proteins, CysC and $\beta 2$ microglobulin $(\beta 2 \mathrm{M})$, and two proteins with high NSC read-through, argonaute-1 (AGO1) and malate dehydrogenase 1 (MDH1).

Gel Analysis of CysC and $\boldsymbol{\beta 2 M}$. Samples were separated on $12 \%$ NUPAGE Bis-Tris gels (catalog number NP0342; Thermo Fisher), alongside a molecular weight standard ranging from 10 to $250 \mathrm{kDa}$ (catalog number 1610373; Bio-Rad Laboratories). Positive control proteins mimicking extended NSC read-through were also generated by using plasmids harboring a point mutation in the NSC for each of the proteins. An in-frame tandem stop codon was present in each case downstream of the NSC. Expression of those plasmids in human embryonic kidney 293 (HEK293) cells gave rise to an elongated form of each of protein, which was then used as the positive control depicting possible read-through products. Sample proteins were transferred from the gels to nitrocellulose membranes (Thermo Fisher) and reacted with primary antibodies against CysC (catalog number 24840; Cell Signaling Technology) or $\beta 2 \mathrm{M}$ (catalog number 12851; Cell Signaling Technology) overnight at $4^{\circ} \mathrm{C}$. Membranes were further incubated with peroxidase-conjugated secondary antibody (catalog number 111-035-045; Jackson ImmunoResearch, West Grove, PA) and developed with the SuperSignal West Pico chemiluminescent substrate (catalog number 34580; Thermo Fisher) by using manual film developing procedure. Ponceau staining (catalog number P7170$1 \mathrm{~L}$; Sigma-Aldrich) of the membranes was used to confirm adequate transfer of protein.

Gel Analysis of AGO1 and MDH1. Samples were heated for 10 minutes at $70^{\circ} \mathrm{C}$ and separated on $7.5 \%$ or $10 \%$ Criterion TGX Stain-Free precast gels (catalog number 5671023 or 5671033 ; Bio-Rad Laboratories). In addition, three dilutions of AGO1 or MDH1 recombinant proteins were separated on each gel. Proteins were transferred to nitrocellulose membranes (catalog number 1704158; Bio-Rad Laboratories) by using the Trans-Blot Turbo Transfer System (Bio-Rad Laboratories). Membranes were incubated in blocking buffer for 1 hour at room temperature and reacted overnight at $4^{\circ} \mathrm{C}$ with primary antibodies against human AGO1 (1:500; catalog number MABE1121; EMD Millipore, Burlington, MA) or MDH1 (1:1000; catalog number PA550446; Thermo Fisher). Membranes were subsequently incubated with peroxidase-conjugated secondary antibody (1:5000; catalog number 111-035-144 or 112-035-062; Jackson ImmunoResearch). Blots were developed with the WesternBright Sirius chemiluminescent substrate (catalog number K-12043-D10; Advansta Inc., San Jose, CA) and photographed with the Aplegen Life Science Omega LumG imager (Gel Company, San Francisco, CA). Ponceau staining (catalog number P7170-1L; Sigma-Aldrich) of the membranes was used to confirm adequate transfer of protein.

\section{PBMC Proteomics}

Cell Treatment and Statistical Analysis. PBMCs from three healthy donors, two separate vials per donor (catalog number CC2702; Lonza, Basel, Switzerland), were thawed, cultured at $37^{\circ} \mathrm{C}$ in $225-\mathrm{cm}^{2}$ cell culture flasks (catalog number CLS3293-25EA; SigmaAldrich) at $5 \times 10^{6}$ cells $/ \mathrm{ml}$, and cultivated in X-Vivo 20 serum-free hematopoetic cell medium (catalog number 04-448Q; Lonza) in duplicates with ELX-02 at concentrations of 0, 20, or $100 \mu \mathrm{g} / \mathrm{ml}$ for 48 hours. These concentrations correspond to the plasma $\mathrm{C}_{\max }$ value and five-times $C_{\max }$, respectively (Leubitz et al., 2019). Samples were washed once in $1 \times \mathrm{PBS}$ and centrifuged at $200 \mathrm{~g}$ for 15 minutes at room 

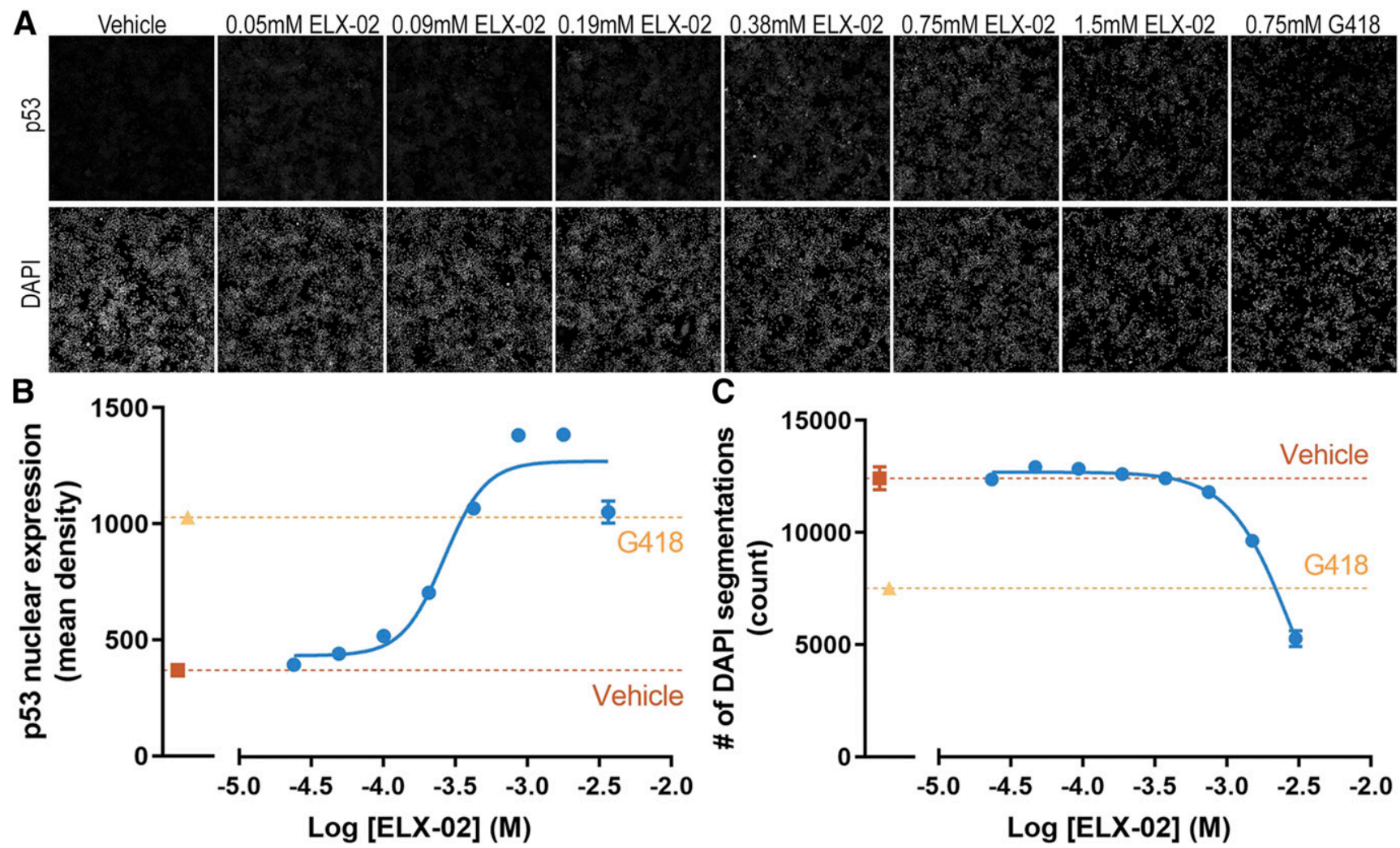

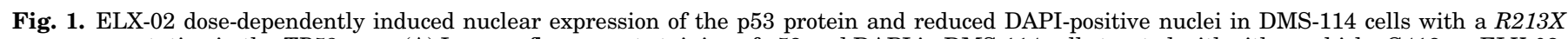

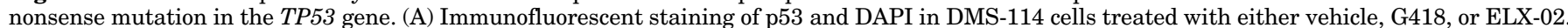

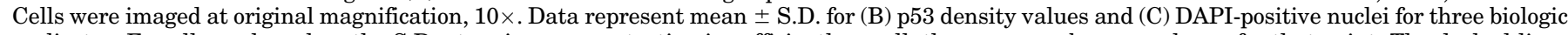

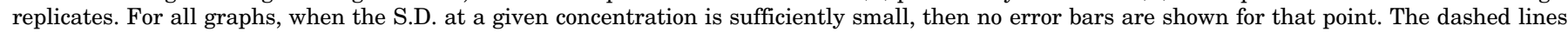

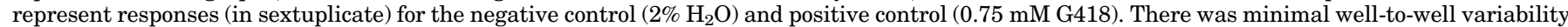

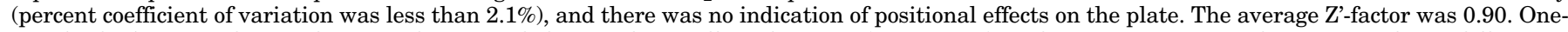

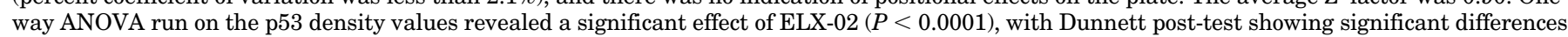
from vehicle at all concentrations of ELX-02 except the lowest.

temperature, and the final cell pellet was snap frozen in dry ice with ethanol. Cell pellets were kept at $-80^{\circ} \mathrm{C}$ until analyses were performed.

PBMC samples were loaded on an Orbitrap Fusion Tribrid mass spectrometer (Thermo Fisher) with a $2 \mathrm{~h} 30$ Reverse Phase gradient and analyzed in Data Dependent Acquisition mode (top 12). The resulting raw files were then searched against the read-through proteome database (Goodenough et al., 2014), with the peptide and protein false discovery rates (FDR) set to $1 \%$. An algorithm was used to match theoretical peptide spectra against observed spectra and assign them a score. To monitor the FDR, a decoy database was generated from the theoretically observable proteome (real search database), and the data were searched against these fake proteins. A score threshold was identified such that the ratio of identifications from the decoy to the real search database was $1 \%$. In addition, each peptide also had a posterior error probability (PEP): the probability that the current identification was erroneous, equal to the modeled local ratio between decoy and real identifications for the peptide's score.

\section{Results}

ELX-02 Produces Significant PSC Read-through of the TP53 Gene of DMS-114 Cells. ELX-02 dosedependently increased the immunofluorescent detection of nuclear p53 protein in DMS-114 cells with an $\mathrm{EC}_{50}$ of $238 \mu \mathrm{M}$ (Fig. 1A), consistent with read-through of the R213X PSC in the TP53 gene and correct nuclear localization of the functional p53 protein. ELX-02 also decreased the number of DAPI-positive nuclei (Fig. 1B). The decreased number of nuclei appears to be related to the increased TP53 readthrough and is consistent with the hypothesis that restoring p53 protein and function in a cancer cell can trigger apoptosis (Mashima et al., 2005; Floquet et al., 2011; Shirole et al., 2016; Bidou et al., 2017). At the lowest concentration of ELX-02 (0.23 $\mathrm{mM}$ ), p53 nuclear expression and DAPI-positive nuclei was not different from vehicle-treated cells. All other concentrations of ELX-02 had p53 nuclear expression that was significantly greater than vehicle-treated cells. High concentrations of ELX-02 (0.75-3 mM) induced p53 nuclear expression levels and loss of DAPI-positive nuclei to an equal or greater degree than G418-treated cells.

ELX-02 increased the truncated and full-length forms of the p53 protein in DMS-114 cells by western blot (Fig. 2, A and B), consistent with read-through of the TP53 gene. Similar results were observed by other groups after G418 treatment of either Calu-6 cells that harbor an R195X mutation in the TP53 gene (Wangen and Green, 2020) or HDQ-P1 cells that harbor an $R 213 X$ mutation in the TP53 gene (Floquet et al., 2011; Bidou et al., 2017). At low concentrations of ELX-02 (0.047 mM), truncated p53 protein could be detected at levels greater than vehicle-treated controls. This increase in truncated p53 protein suggests small amounts of PSC read-through likely occurred, which then may have removed exon junction 


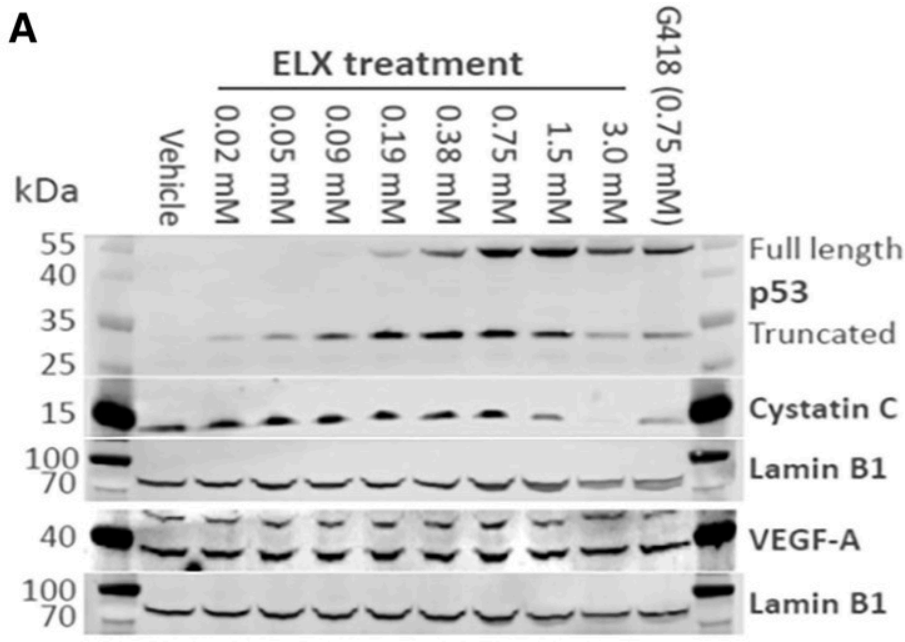

B

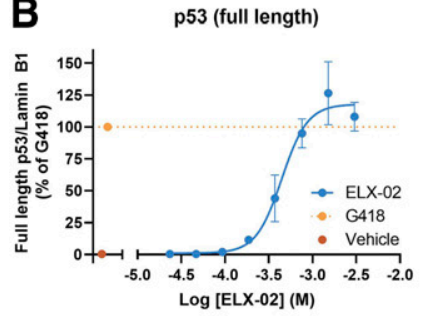

C

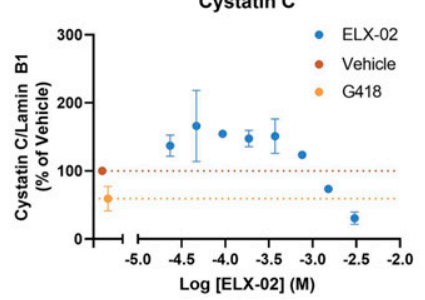

p53 (truncated)

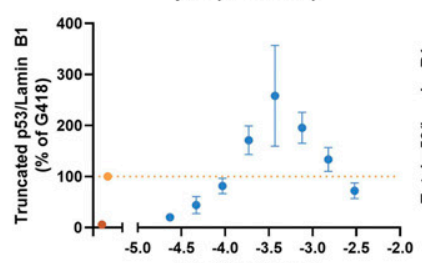

$\log [\mathrm{ELX}-02]$ (M)
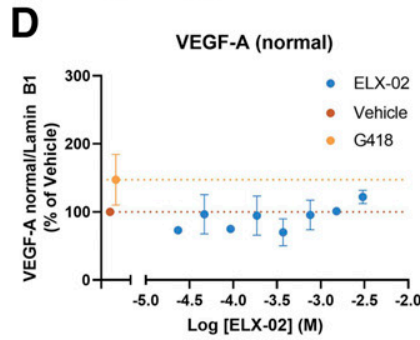

p53 (total)

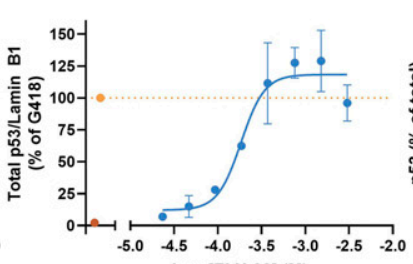

Log [ELX-02] (M)

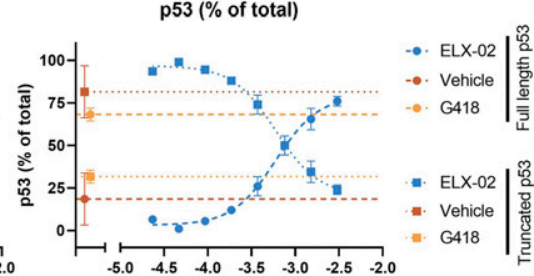

Log [ELX-02] (M)

Fig. 2. ELX-02 dose-dependently increased both truncated and full-length p53 protein while having no effect on CysC or VEGF-A expression. (A) Western blot depicting detection of either truncated (band at $\sim 34 \mathrm{kDa}$ ) or full-length (band at $\sim 53 \mathrm{kDa}$ ) p53 protein. Preliminary experiments with recombinant protein indicated a lower limit of detection at $1.6 \mathrm{ng}$ of p53 protein. Blots were also probed to detect Lamin B1 (band at $\sim 70 \mathrm{kDa}$ ), CysC (band at $\sim 13 \mathrm{kDa}$ ), and VEGF-A (bands at $\sim 38 \mathrm{kDa}$ for normal protein and at $\sim 43 \mathrm{kDa}$ for NSC read-through protein). Lower limits of detection of $0.6 \mathrm{ng}$ CysC and $10 \mathrm{ng}$ VEGF-A were established by using recombinant proteins. (B) Data represent mean \pm S.D. p53 protein intensity values for the biologic duplicates, expressed as a percentage of the G418 effect in the first three panels or as the percent of total protein in the last panel. One-way ANOVA run on the full-length p53 protein levels revealed a significant effect of ELX-02 $(P<0.0001)$, with Dunnett post-test showing significant differences from vehicle at ELX-02 concentrations of $0.38 \mathrm{mM}$ and higher. (C) Data represent mean \pm S.D. CysC protein intensity values for the biologic duplicates. (D) Data represent mean \pm S.D. VEGF-A protein intensity values for the biologic duplicates, with attention paid to levels of the normal protein product, the longer NSC read-through protein product, and total VEGF-A protein.

complex proteins and led to less NMD, greater mRNA stability, and production of more protein overall (Keeling et al., 2014). The increase in truncated p53 protein by ELX02 peaked at $0.38 \mathrm{mM}$ and then dropped off as ELX-02 effectively induced read-through of the PSC in TP53 mRNA and generated full-length p53 protein. Full-length p53 protein could be detected by using ELX-02 concentrations as low as $0.09 \mathrm{mM}$, with concentrations of $0.38 \mathrm{mM}$ and higher showing protein amounts significantly greater than vehicle. Higher concentrations of ELX-02 produced more full-length p53 protein, with an $\mathrm{EC}_{50}$ of $446 \mu \mathrm{M}$ and peak efficacy greater than or equal to what was observed for G418. The percentage of full-length and truncated p53 proteins at high concentrations of ELX-02 were similar to those observed with G418 treatment.

In samples taken from the same DMS-114 cells, the effect of ELX-02 on read-through of two other genes was tested: 1)
CysC (a $\sim 13 \mathrm{kDa}$ housekeeping gene, which is a reversible competitive inhibitor of papain-like cysteine proteases) and 2) VEGF-A (a dimeric glycoprotein that induces growth of blood vessels, with weights of $\sim 38 \mathrm{kDa}$ for normal protein and at $\sim 43 \mathrm{kDa}$ for NSC read-through protein). It was hypothesized that proteins showing an increase in NSC read-through by ELX-02 would be proteins with high expression across all cells or proteins with basal levels of native read-through higher than other proteins. A decrease in the amount of CysC protein at high concentrations of ELX-02 was observed (Fig. 2, A and C). This effect may be due to cellular toxicity at high ELX-02 concentrations that impact CysC earlier than the other housekeeping gene Lamin B1, potentially because of differences in the half-life of each protein. No high molecular weight CysC protein product from NSC read-through could be detected. Additionally, there was no effect of ELX-02 on the expression of VEGF-A (Fig. 2, A and D) when measuring either 

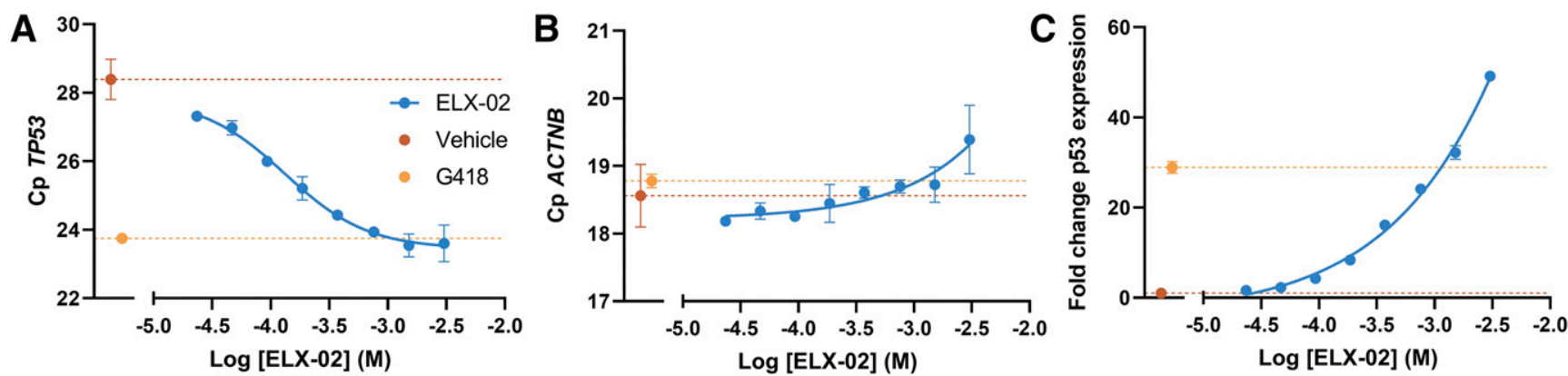

Fig. 3. ELX-02 dose-dependently increased TP53 transcript levels. RNA samples were of satisfactory quality to perform quantitative polymerase chain reaction (concentration $>115 \mathrm{ng} / \mu \mathrm{l}$; A260/A280 > 1.8; A260/A230 > 1.3). Data represent mean \pm S.D. Cp values for the technical triplicates for (A) TP53 or (B) ACTNB. (C) The fold change increase for TP53 mRNA was calculated by using the relative Cp values for both TP53 and ACTNB. One-way ANOVA run on the TP53 transcript level values revealed a significant effect of ELX-02 $(P<0.0001)$, with Dunnett post-test showing significant differences from vehicle at all concentrations of ELX-02 except the lowest.

the standard-length protein or the higher molecular weight protein that would result from NSC read-through.

Finally, ELX-02 dose-dependently decreased the Cp value for TP53 mRNA with an $\mathrm{IC}_{50}$ of $133 \mu \mathrm{M}$, indicating an increase in TP53 steady-state transcript levels in DMS-114 cells (Fig. 3A). This result is consistent with western blot data, suggesting decreased NMD of the TP53 mRNA product resulting in increased protein levels. Alternatively, the increased transcript and protein levels could indicate a direct effect of ELX-02 that produces increased expression from the TP53 gene. High concentrations of ELX-02 resulted in a Cp that was similar to G418-treated cells. ELX-02 had no effect on the $\mathrm{Cp}$ value for the housekeeping gene for $\beta$-actin, $A C T N B$, until the highest dose of $3 \mathrm{mM}$ (Fig. 3B), suggesting toxicity at the highest dose. By using the relative Cp values for both TP53 and $A C T N B$, the fold change in expression for TP53 was calculated. Here, after disregarding the highest concentration that is confounded by cellular toxicity, ELX-02 treatment resulted in $>30$-fold increase in TP53 transcript levels relative to vehicle controls (Fig. 3C), which is similar to the effect observed with G418 treatment. Furthermore, these findings are consistent with results obtained from treating Calu- 6 cells with G418, wherein a 16-fold increase in TP53 mRNA levels was observed (Wangen and Green, 2020).

No Evidence of Native Stop Codon Read-Through in Ex Vivo Clinical Samples. NSC read-through was examined for the housekeeping protein CysC in PBMCs from all clinical trial subjects treated with either placebo or ELX-02. Additionally, native read-through of $\beta 2 \mathrm{M}$, a component of major histocompatibility complex class I molecules present on all nucleated cells, was examined following treatment of ELX-02. $\mathrm{Cys} \mathrm{C}$ and $\beta 2 \mathrm{M}$ each showed one band at the expected molecular weight for the native protein $(\sim 13$ and $\sim 12 \mathrm{kDa}$ for CysC and $\beta 2 \mathrm{M}$, respectively; representative blot from subjects dosed with $5.0 \mathrm{mg} / \mathrm{kg}$ ELX-02 are provided in Fig. 4). The higher molecular weight species for these two proteins were not observed in any of the clinical samples, indicating no measurable NSC readthrough translation of these housekeeping genes and no production of elongated protein variants.

PBMCs from two cohorts of clinical trial test subjects (placebo and $7.5 \mathrm{mg} / \mathrm{kg}$ ELX-02) were also analyzed for readthrough of genes with high basal NSC read-through. AGO1 (a $90 \mathrm{kDa}$ protein that plays a central role in RNA silencing processes) and MDH1 (a $35 \mathrm{kDa}$ enzyme that catalyzes the reversible oxidation of malate to oxaloacetate) each use a native Opal, UGA, stop codon, which is most susceptible to both NSC read-through and compound-induced PSC readthrough (Cridge et al., 2018). We chose to focus on the 7.5$\mathrm{mg} / \mathrm{kg}$ ELX-02 group because this dose level provided the highest clinical exposure to ELX-02 (Leubitz et al., 2019) and therefore would be most likely to induce NSC read-through. Samples showed expression of the native AGO1 and MDH1 proteins at the expected molecular weights (representative blots from subjects receiving either placebo or $7.5 \mathrm{mg} / \mathrm{kg}$ ELX02 are provided in Fig. 5). The native AGO1 signal was lower than the limit of quantification (1.5 ng recombinant AGO1) and at the limit of detection. Basal read-through appeared to be minimal for this protein and no noticeable increase was

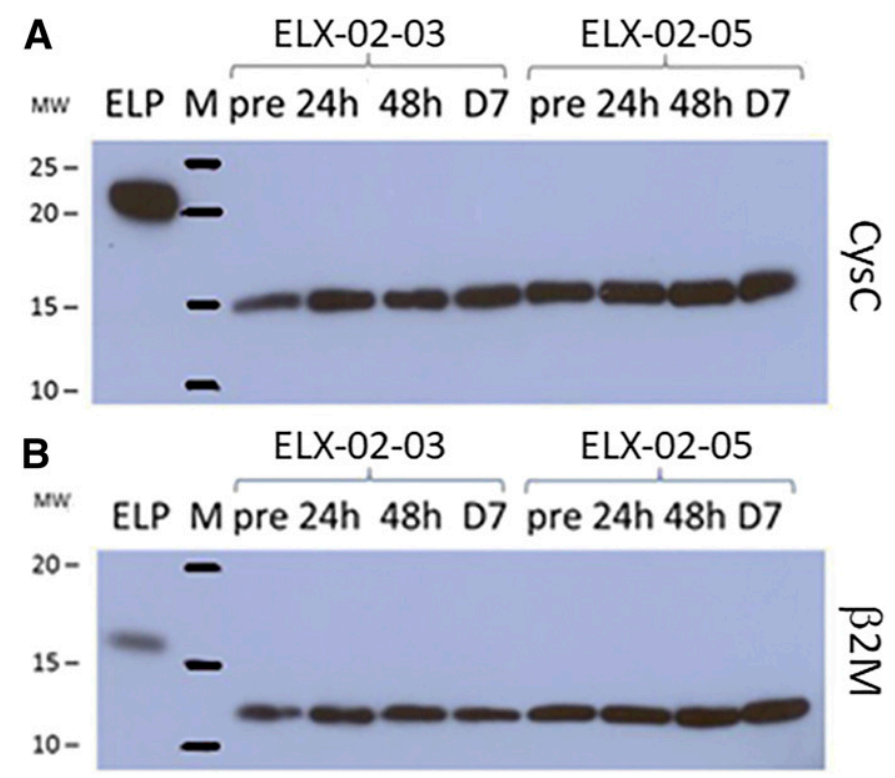

Fig. 4. Representative western blot staining of $\mathrm{CysC}$ and $\beta 2 \mathrm{M}$ for samples from two subjects administered ELX-02 at $5.0 \mathrm{mg} / \mathrm{kg}$. (A) A single band of $\sim 15 \mathrm{kDa}$ is observed for CysC, consistent with the $13 \mathrm{kDa}$ native form of the protein. (B) A band of $\sim 12 \mathrm{kDa}$ is observed for $\beta 2 \mathrm{M}$, also consistent with the native form of the protein. No read-through product is observed for either CysC or $\beta 2 \mathrm{M}$ at either predose baseline or in response to ELX-02 in this blot and in blots from other subjects dosed with ELX-02. Samples were collected from clinical study EL-001. Codes above the blots refer to the patient identification number used to track the four samples collected from each trial participant. $24 \mathrm{~h}$ and $48 \mathrm{~h}$, samples taken 24 and 48 hours after ELX-02 treatment; D7, samples taken 7 days after ELX-02 treatment; ELP, elongated protein positive control; M, MW marker; MW, molecular weight; pre, predose. 


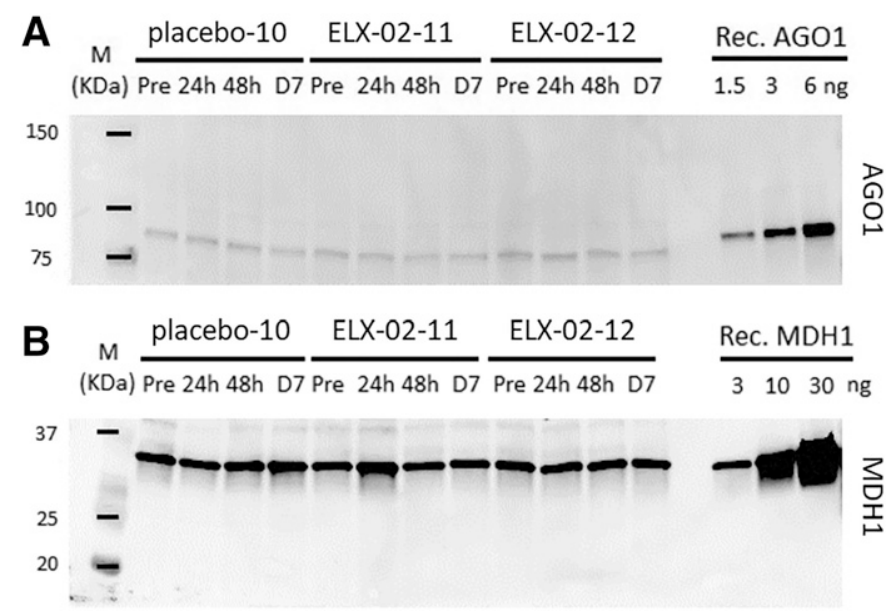

Fig. 5. Representative western blot staining of AGO1 and MDH1 for samples from subjects administered either placebo or ELX-02 at $7.5 \mathrm{mg} / \mathrm{kg}$. (A) A single band of $90 \mathrm{kDa}$ is observed for AGO1, consistent with the native form of the protein. Read-through of the NSC for AGO1 would add 33 amino acids to the protein and result in a molecular weight of roughly $94 \mathrm{kDa}$. However, no read-through product is observed for AGO1. (B) A band of $35 \mathrm{kDa}$ is observed for MDH1. Read-through of the NSC for MDH1 would add 19 amino acids and result in a molecular weight of roughly 37 $\mathrm{kDa}$. A faint band immediately above the primary band may represent this read-through product. Additional bands of higher molecular weight above these bands likely represent nonspecific protein recognition by the antibody. Recombinant proteins were run alongside each set of samples and provide information as to the lower limit of detection in each western blot (1.5 and $3 \mathrm{ng}$ for $\mathrm{AGO} 1$ and $\mathrm{MDH} 1$, respectively). Samples were collected from clinical study EL-006. Codes above the blots refer to the patient identification number used to track the four samples collected from each trial participant. 24h and 48h, samples taken 24 and 48 hours after ELX-02 treatment; D7, samples taken 7 days after ELX-02 treatment; M, MW marker; MW, molecular weight; pre, predose.

observed in samples collected after receiving ELX-02. The native $\mathrm{MDH} 1$ signal was within the range of quantitation (3 ng recombinant MDH1). Though a faint band consistent with a read-through product could be observed immediately above the primary band for $\mathrm{MDH} 1$, the basal read-through levels of this protein were below the limit of detection. ELX-02 treatment had no effect on the intensity of this potential MDH1 read-through band. These results with AGO1 and $\mathrm{MDH} 1$, alongside results with $\mathrm{CysC}$ and $\beta 2 \mathrm{M}$, therefore do not provide evidence for NSC read-through proteins in ex vivo samples.

Proteomic Analysis of ELX-02 Effect on PBMCs. To explore the possibility of NSC read-through by ELX-02 using an unbiased proteomics approach, a read-through peptide database was generated from the National Center for Biotechnology Information RefSeq Database. In silico digestion of the real database generates about 1,895,000 unique peptide sequences. To monitor the FDR, a decoy database was generated from the theoretically observable proteome, which generates an additional 1,527,000 unique peptides (not contained in the former). A total of 72,661 peptides were identified in ELX-02 and control-treated PBMC extracts, corresponding to 49,133 modified peptide sequences. These were mapped to 6086 protein group identifiers. The mass spectrometry results were subsequently searched against our custom RefSeq database of human proteome sequences that include sequences at and after the NSC of each gene to identify readthrough-derived peptides while excluding masses that had matched within conventional coding regions.

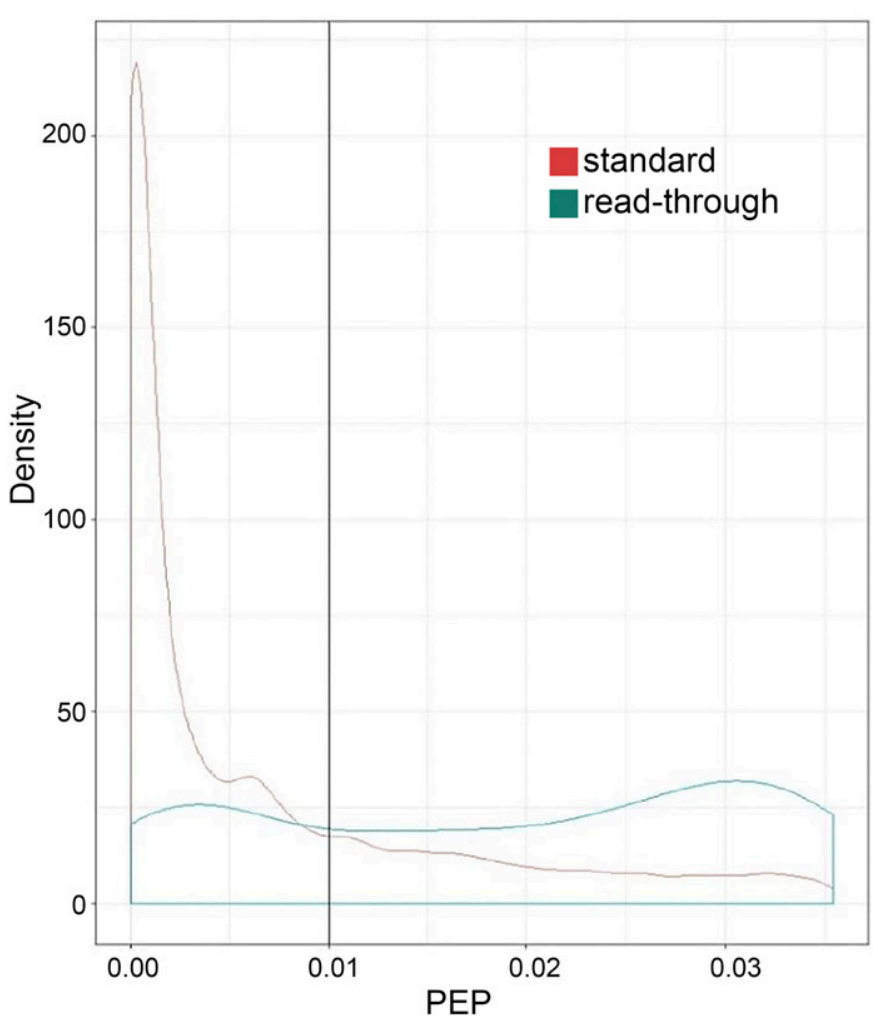

Fig. 6. Distribution of PEPs for identified standard (red) and readthrough (blue) peptides. Read-through peptides tend to have higher PEPs than standard peptides, which is consistent with the idea that a high proportion of identified read-through peptides are false hits.

The number of false discoveries expected from the readthrough database of human transcriptome was determined by calculating the number of peptides in the real and readthrough databases. To identify true read-through peptides, the peptide and protein FDR was set to $1 \%$, which translates to an expectation of about 491 of the peptides identified in the real database to be false hits. By scaling for the respective sizes of the peptide ensembles, we predicted an approximate number of false discoveries from the read-through database of about 396. These calculations are based on the assumption that, on average, the chemistry of both peptide ensembles is similar. Our analysis demonstrates that the number of readthrough sequences in ELX-02-treated samples (124 peptides) was lower than the predicted FDR levels of read-through peptides (396 peptides). The distribution of PEPs (Fig. 6), the probability that there is an error in identification of standard (red) or read-through (blue) peptides, indicates that readthrough peptides tend to have higher PEPs than standard peptides, consistent with the conclusion that a high proportion of identified read-through peptides are false hits.

To estimate if any of the identified 124 peptides correspond to an actual read-through peptide, we used a bootstrapping strategy to estimate the probability distribution of random samples of 124 peptides picked from the standard distribution. The mean of the median of 1,000,000 samplings of 124 PEP values from non-read-through standard peptides identified in our experiment was 0.0021 . The median PEP for the 124 identified read-through peptides was approximately 0.020 (0.00079), 10-times higher than the standard peptides mean. Thus, we have a population of read-through peptides with 
a significantly higher PEP than expected for the population of similarly sized samples, suggesting that at least some of the read-through peptides represent actual read-through. The 124 peptides map to 108 different proteins. Of those 108 proteins, only seven proteins were identified with more than a single peptide, indicating a higher confidence of read-through detection (Supplemental Table 1). To test if any of the detected read-through peptides in ELX-02-treated samples are significant, we used the Benjamini Hochberg FDR procedure to analyze $P$ values by using a high-accepted FDR of $30 \%$. The seven proteins with more than one peptide identification were detected in both control and ELX-02 treated cells. Signal intensities were not statistically different between control and ELX-02 treated cells, indicating that these proteins undergo natural read-through. Thirty-nine read-through peptides were identified only in control cells (Supplemental Table 1, $P$ value $=$ not applicable). At the lower ELX-02 concentration $(20 \mu \mathrm{g} / \mathrm{ml})$, none of the $P$ values showed statistically significant change from control cells, whereas only one protein showed statistical significance at $100 \mu \mathrm{g} / \mathrm{ml}$. With FDR set at $30 \%$, there is a $30 \%$ chance of this hit being a false discovery. Therefore, these proteomics results do not provide strong evidence for induction of NSC read-through by ELX-02 in human PBMCs.

\section{Discussion}

The studies reported here demonstrate that ELX-02 produced significant PSC read-through of an exemplar nonsense mutation in the TP53 gene of a cancer cell line but did not increase the presence of extended protein variants indicative of NSC read-through in either DMS-114 cells or PBMCs derived from normal healthy human subjects. These studies build on previous work testing the potential for translational read-through of nonsense mutations by ELX-02 using in vitro models (Kandasamy et al., 2012; Xue et al., 2014; Bidou et al., 2017; Friesen et al., 2018; Brasell et al., 2019) and in vivo mouse models of cystic fibrosis and cystinosis (Xue et al., 2014; Brasell et al., 2019). Collectively, these studies show that ELX-02 restores functional activity to proteins encoded by genes containing a PSC yet has limited impact on NSCs and supports its therapeutic potential in diseases resulting from nonsense alleles.

Given that nonsense mutations comprise a significant fraction of all alleles causing inherited disorders (Krawczak et al., 2000; Mort et al., 2008), drugs that promote PSC readthrough could have broad therapeutic potential. Translational read-through-inducing drugs such as ELX-02 work by suppressing the recognition of nonsense mutations by translation termination machinery, thereby allowing insertion of nearcognate amino acids and production of functional full-length proteins. However, one aspect of tolerability for therapeutic nonsense suppression is the assurance that the compound does not suppress NSCs and generate aberrant proteins. It was hypothesized that if any proteins would show an increase in NSC read-through as a result of ELX-02 exposure, it would be proteins showing high expression levels across all cells or proteins with basal levels of NSC read-through that are higher than average. We found that ELX-02 does not generate or increase extended protein variants that would be consistent with NSC read-through of housekeeping genes (CysC and $\beta 2 \mathrm{M}$ ) or high native read-through genes (VEGF-A, AGO1, and MDH1).

As targeted efforts to detect NSC read-through did not detect evidence of such activity, we undertook a proteomic approach to provide unbiased detection of read-through peptides for most human-expressed proteins. Our analysis demonstrates that the number of read-through sequences in ELX-02-treated samples was lower than the predicted FDR levels of read-through peptides. Read-through peptides were also observed to have higher PEP distributions than standard peptides, consistent with the conclusion that a high proportion of read-through peptides were false hits. Only seven proteins were identified with more than one peptide, which would be a signal for higher confidence in potential read-through. These peptides were observed in both control and ELX-02-treated samples, and the signal intensity was not significantly different between control and treatment groups. Also, with only one protein with one peptide assignment showing a statistically higher intensity at 100 versus $20 \mu \mathrm{g} / \mathrm{ml}$ ELX02, in general, no dose-dependent response was observed. Because this top ELX-02 concentration corresponds to fivefold $\mathrm{C}_{\max }$ observed at the highest dose administered in a clinical study, these results suggest that ELX-02 does not induce general read-through at clinically relevant doses.

Recent work has similarly tested whether G418 induces stop codon read-through in either HEK293 cells carrying a reporter construct or Calu-6 cells that harbor an $R 195 X$ mutation in the TP53 gene (Wangen and Green, 2020). By using ribosome profiling, they observed PSC read-through by G418, which was influenced by the stop codon identity and the surrounding mRNA sequence context, similar to what has been established in the read-through field (Floquet et al., 2011; Dabrowski et al., 2018). They also found that the majority of mRNAs were neither dramatically changed in abundance nor differentially translated in response to G418, but significant NSC read-through by G418 led to substantial effects on translation of histone genes, selenoprotein genes, and S-adenosylmethionine decarboxylase. Although this effect is worth attention, other work has found that translation past NSCs into the $3^{\prime}$ untranslated region may encode peptide sequences that destabilize the attached protein, providing mitigation of unwelcome and varied translation errors (Arribere et al., 2016). This destabilization could explain the increased unfolded protein response observed in Calu- 6 cells (Wangen and Green, 2020) as well as the lack of extended protein variants in our studies using western blot detection of in vitro and ex vivo samples. Together, these data imply that ELX-02 could promote read-through at NSCs, but that evolutionary pressure has put cis-acting elements into place to limit NSC read-through and that cellular processes exist to provide efficient clearance of those potential NSC readthrough protein products, thus limiting their abundance below detection limits.

In conclusion, ELX-02 produces significant read-through of PSCs leading to the production of full-length functional protein, whereas three complementary techniques suggest that ELX-02 does not promote read-through of NSCs. Thus, ELX02 may be a potential therapeutic option for nonsense mutation-mediated genetic diseases. Though our results suggest that ELX-02 does not promote read-through of NSCs, further studies across additional genes and protein products are needed. 


\section{Acknowledgments}

The authors would like to thank Esmee Koedoot, Sabrina de Munnik, Sanne Holt, and Folkert Verkaar at Charles River Laboratories Leiden for the DMS-114 cell line work; Raphael Mayer at Smart Assays Biotechnologies for the clinical sample work; and Donald Smith at DC Biosciences for the proteomics work.

\section{Authorship Contributions}

Participated in research design: Crawford, Alroy, Sharpe, Goddeeris.

Conducted experiments: Crawford, Alroy.

Performed data analysis: Crawford, Alroy.

Wrote or contributed to the writing of the manuscript: Crawford, Alroy, Sharpe, Goddeeris, Williams.

\section{References}

Arribere JA, Cenik ES, Jain N, Hess GT, Lee CH, Bassik MC, and Fire AZ (2016) Translation readthrough mitigation. Nature 534:719-723.

Bidou L, Bugaud O, Belakhov V, Baasov T, and Namy O (2017) Characterization of new-generation aminoglycoside promoting premature termination codon readthrough in cancer cells. RNA Biol 14:378-388.

Bordeira-Carriço R, Pêgo AP, Santos M, and Oliveira C (2012) Cancer syndromes and therapy by stop-codon readthrough. Trends Mol Med 18:667-678.

Brasell EJ, Chu LL, Akpa MM, Eshkar-Oren I, Alroy I, Corsini R, Gilfix BM, Yamanaka Y, Huertas P, and Goodyer P (2019) The novel aminoglycoside, ELX-02, permits CTNSW138X translational read-through and restores lysosomal cystine efflux in cystinosis. PLoS One 14:e0223954.

Burke JF and Mogg AE (1985) Suppression of a nonsense mutation in mammalian cells in vivo by the aminoglycoside antibiotics G-418 and paromomycin. Nucleic Acids Res 13:6265-6272.

Celik A, Kervestin S, and Jacobson A (2015) NMD: at the crossroads between translation termination and ribosome recycling. Biochimie 114:2-9.

Clancy JP, Bebök Z, Ruiz F, King C, Jones J, Walker L, Greer H, Hong J, Wing L, Macaluso M, et al. (2001) Evidence that systemic gentamicin suppresses premature stop mutations in patients with cystic fibrosis. Am J Respir Crit Care Med 163:1683-1692.

Cridge AG, Crowe-McAuliffe C, Mathew SF, and Tate WP (2018) Eukaryotic translational termination efficiency is influenced by the $3^{\prime}$ nucleotides within the ribosomal mRNA channel. Nucleic Acids Res 46:1927-1944.

Dabrowski M, Bukowy-Bieryllo Z, and Zietkiewicz E (2018) Advances in therapeutic use of a drug-stimulated translational readthrough of premature termination codons. Mol Med $\mathbf{2 4} \cdot 25$.

Floquet C, Deforges J, Rousset JP, and Bidou L (2011) Rescue of non-sense mutated p53 tumor suppressor gene by aminoglycosides. Nucleic Acids Res 39:3350-3362.

Fourmy D, Yoshizawa S, and Puglisi JD (1998) Paromomycin binding induces a local conformational change in the A-site of 16 S rRNA. J Mol Biol 277:333-345.

Friesen WJ, Johnson B, Sierra J, Zhuo J, Vazirani P, Xue X, Tomizawa Y, Baiazitov $\mathrm{R}$, Morrill C, Ren H, et al. (2018) The minor gentamicin complex component, X2, is a potent premature stop codon readthrough molecule with therapeutic potential. PLoS One 13:e206158.

Frischmeyer PA and Dietz HC (1999) Nonsense-mediated mRNA decay in health and disease. Hum Mol Genet 8:1893-1900.

Goodenough E, Robinson TM, Zook MB, Flanigan KM, Atkins JF, Howard MT, and Eisenlohr LC (2014) Cryptic MHC class I-binding peptides are revealed by aminoglycoside-induced stop codon read-through into the $3^{\prime}$ UTR. Proc Natl Acad Sci USA 111:5670-5675.

Kandasamy J, Atia-Glikin D, Shulman E, Shapira K, Shavit M, Belakhov V, and Baasov T (2012) Increased selectivity toward cytoplasmic versus mitochondrial ribosome confers improved efficiency of synthetic aminoglycosides in fixing damaged genes: a strategy for treatment of genetic diseases caused by nonsense mutations. J Med Chem 55:10630-10643.

Keeling KM, Xue X, Gunn G, and Bedwell DM (2014) Therapeutics based on stop codon readthrough. Annu Rev Genomics Hum Genet 15:371-394.

Krawczak M, Ball EV, Fenton I, Stenson PD, Abeysinghe S, Thomas N, and Cooper DN (2000) Human gene mutation database-a biomedical information and research resource. Hum Mutat 15:45-51.

Leubitz A, Frydman-Marom A, Sharpe N, van Duzer J, Campbell KCM, and Vanhoutte F (2019) Safety, tolerability, and pharmacokinetics of single ascending doses of ELX-02, a potential treatment for genetic disorders caused by nonsense mutations, in healthy volunteers. Clin Pharmacol Drug Dev 8:984-994.

Linde L and Kerem B (2008) Introducing sense into nonsense in treatments of human genetic diseases. Trends Genet 24:552-563.

Mashima T, Oh-hara T, Sato S, Mochizuki M, Sugimoto Y, Yamazaki K, Hamada J, Tada M, Moriuchi T, Ishikawa Y, et al. (2005) p53-defective tumors with a functional apoptosome-mediated pathway: a new therapeutic target. $J$ Natl Cancer Inst 97:765-777.

Miller JN and Pearce DA (2014) Nonsense-mediated decay in genetic disease: friend or foe? Mutat Res Rev Mutat Res 762:52-64.

Moazed D, Stern S, and Noller HF (1986) Rapid chemical probing of conformation in $16 \mathrm{~S}$ ribosomal RNA and $30 \mathrm{~S}$ ribosomal subunits using primer extension. $J \mathrm{Mol}$ Biol 187:399-416.

Mort M, Ivanov D, Cooper DN, and Chuzhanova NA (2008) A meta-analysis of nonsense mutations causing human genetic disease. Hum Mutat 29:1037-1047.

O'Sullivan ME, Perez A, Lin R, Sajjadi A, Ricci AJ, and Cheng AG (2017) Towards the prevention of aminoglycoside-related hearing loss. Front Cell Neurosci 11:325

Pokrovskaya V, Nudelman I, Kandasamy J, and Baasov T (2010) Aminoglycosides redesign strategies for improved antibiotics and compounds for treatment of human genetic diseases. Methods Enzymol 478:437-462.

Prayle A, Watson A, Fortnum H, and Smyth A (2010) Side effects of aminoglycosides on the kidney, ear and balance in cystic fibrosis. Thorax 65:654-658.

Prokhorova I, Altman RB, Djumagulov M, Shrestha JP, Urzhumtsev A, Ferguson A Chang CT, Yusupov M, Blanchard SC, and Yusupova G (2017) Aminoglycoside interactions and impacts on the eukaryotic ribosome. Proc Natl Acad Sci USA 114: E10899-E10908.

Sabbavarapu NM, Pieńko T, Zalman BH, Trylska J, and Baasov T (2018) Exploring eukaryotic versus prokaryotic ribosomal RNA recognition with aminoglycoside derivatives. MedChemComm 9:503-508.

Schuller AP and Green R (2018) Roadblocks and resolutions in eukaryotic translation. Nat Rev Mol Cell Biol 19:526-541.

Shalev M and Baasov T (2014) When proteins start to make sense: fine-tuning aminoglycosides for PTC suppression therapy. MedChemComm 5:1092-1105.

Shirole NH, Pal D, Kastenhuber ER, Senturk S, Boroda J, Pisterzi P, Miller M, Munoz G, Anderluh M, Ladanyi M, et al. (2016) TP53 exon-6 truncating mutations produce separation of function isoforms with pro-tumorigenic functions [published correction appears in eLife (2017) 6]. eLife $\mathbf{5}$.

Shulman E, Belakhov V, Wei G, Kendall A, Meyron-Holtz EG, Ben-Shachar D, Schacht J, and Baasov T (2014) Designer aminoglycosides that selectively inhibit cytoplasmic rather than mitochondrial ribosomes show decreased ototoxicity: a strategy for the treatment of genetic diseases. J Biol Chem 289:2318-2330.

Wangen JR and Green R (2020) Stop codon context influences genome-wide stimulation of termination codon readthrough by aminoglycosides. eLife $\mathbf{9}$

Wilschanski M, Yahav Y, Yaacov Y, Blau H, Bentur L, Rivlin J, Aviram M, BdolahAbram T, Bebok Z, Shushi L, et al. (2003) Gentamicin-induced correction of CFTR function in patients with cystic fibrosis and CFTR stop mutations. $N$ Engl J Med 349:1433-1441.

Xue X, Mutyam V, Tang L, Biswas S, Du M, Jackson LA, Dai Y, Belakhov V, Shalev M, Chen F, et al. (2014) Synthetic aminoglycosides efficiently suppress cystic fibrosis transmembrane conductance regulator nonsense mutations and are enhanced by ivacaftor. Am J Respir Cell Mol Biol 50:805-816.

Address correspondence to: Matthew M. Goddeeris, Eloxx Pharmaceuticals, Inc., 950 Winter Street, Waltham, MA 02451. E-mail: matthew.goddeeris@eloxxpharma.com 